\title{
Refibrillation during out-of-hospital arrest: A frequent event with clinical consequences
}

RUDOLPH W. KOSTER ( $\square)$ Department of Cardiology, room F3-239 Academic Medical Center Meibergdreef 9 1105 AZ Amsterdam The Netherlands Phone: +31-20-5662608 E-mail: r.w.koster@amc.nl

\author{
RUDOLPH W. KOSTER
}

\begin{abstract}
The refibrillation was a frequent event in out-of-hospital cardiac arrest (OHCA). The number of recurrences of ventricular fibrillation (VF) is in inverse relationship with survival. In this article we discuss about causes and mechanism of refibrillation. The amiodarone and new technical solution (defibrillators that may allow continuous monitoring of the heart rhythm, while chest compressions continue and recommend defibrillation when refibrillation occurs) are promising new strategy to improve outcome of OHCA and recurrent VF.
\end{abstract}

Keywords: out-of-hospital cardiac arrest, ventricular fibrillation, defibrillation, refibrillation, amiodarone, continuous monitoring of the heart rhythm

\section{Introduction \\ If one reviews cases of out-of-hospital cardiac arrest (OHCA) and the monito- red recording of the resuscitation effort in full, the first focus is on the initial rhythm. If ventricular fibrillation (VF) is seen, a defibrillation shock is given and VF removed, interest is on the rhythm that follows. Mostly, initially there is asystole, occasionally immediately an organised -but not immediately perfusing- rhythm. When the recording continues, all three possibilities of rhythm can be seen: con- tinued asystole, slow escape rhythms, picking up rate or recurrence of VF, sometimes from asystole without iden- tified rhythm prior to recurrence or from an organised rhythm with or without a new initiation premature beat. After another shock, this sequence may recur more than once.}

\section{Incidence and prognosis}

Only with full and continuous recordin- gs of a series of cardiac arrest cases we can reliably describe the rate of recurrence of VF. One of the first to describe the incidence is White et al. (1) who observed it in 29 of 49 patients $(61 \%)$, while $35 \%$ had more than one recurrence. Their information was limited to automatic external defibrillator (AED) recordings and they found no relation with return of spontaneous circulation (ROSC) or survival of the patients. Shortly thereafter, we also analysed recordings from $\mathrm{OHCA}$ in a larger patient experience and including the full prehospital resuscitation from AED and Emergency Medical Service (EMS) defibrillator recordings, merging data from both, when appliccable. (2) Our study confirmed that recurrence of VF was a frequent event: we observed it in $79 \%$ of 322 of patients, while a quarter of patients refibrillated more than 4 times. Recurrence occurred median 45 seconds after defibrillation. In contrast to White, we found a negative correlation between the number of recurrences and survival. We could not identify an association between any patient or resuscitation characteristic and recurrence of VF.
Recently, in a new large dataset we confirmed the relation between the number of recurrences of VF and survival, but also measured the total duration of all recurrences cumulatively - "VF burden" and found a similar inverse relationship with survival. (unpublished observations) This suggests that not only prevention of refibrillation may be important, but also to limit the duration of each episode.

\section{Causes of refibrillation}

In general, VF in the context of myocardial ischaemia is caused by ventricular premature beats, that initiate a re-entry circuit of Ventricular Tachycardia, splitting up in smaller circuits that break up in the small re-entry circuits that characterize VF. The mode of initiation of recurrence of VF is not always straightforward. Sometimes it clearly follows the described mechanism when an organised rhythm is interrupted by a ventricular premature beat (figure 1A). Other times, chaotic ventricular rhythm with multiform premature beats deterioriate into VF (figure 1B). Sometimes, $V F$ recurs from asystole, where the first ventricular depolarisation results in VF, 
A

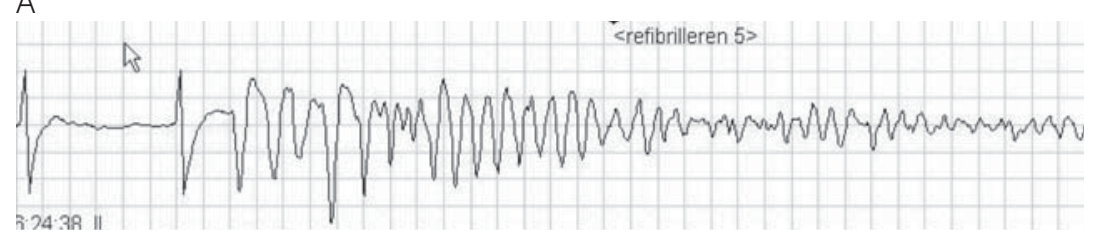

B

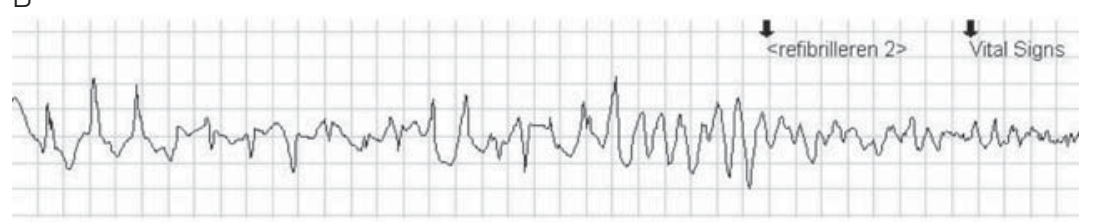

C

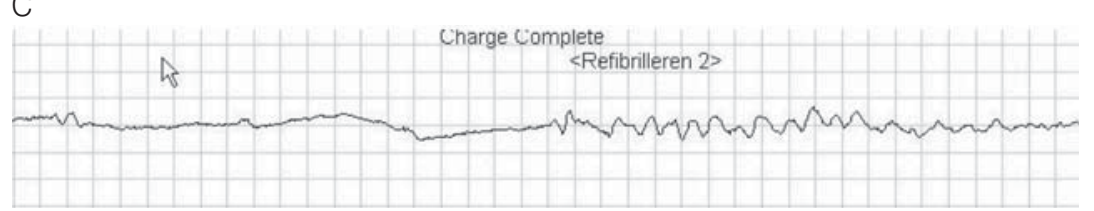

Figure 1. Three examples of monitored cases of Out-of-Hospital Cardiac Arrest and the various ways refibrillation can be initiated.

and a clear initiating premature beat cannot be identified (figure 1C).

Another point of interest is the question if chest compressions from cardiopulmonary resuscitation (CPR) can induce premature beats and initiate recurrence of VF. This was denied earlier by Hess and White (3) in a series of 35 patients with recurrences. They found an equal occurrence with or without chest compressions, but they were not able to make a clear association with the exact start of chest compressions. Such an association was first found in an animal experiment by Osorio, (4) who suggested that the mechanical effect of the chest compression initiated the electrical premature beat that initiated recurrent VF. We were able to demonstrate the same phenomenon in a clinical trial, in which in half the patients chest compressions were resumed immediately (median 8 seconds) after defibrillation, corresponding with the recommendations of the Guidelines for resuscitation, issued in 2005. In the other half of the patients, after defibrillation first the rhythm was checked, then a check for pulsations was performed, and only then chest compressions resumed when indicated, a median 30 seconds after defibrillation, according to the also because the recurrence itself may be influenced by amiodarone, nbut ot the duration or each recurrence. Therefore not only new strategies need to be developed to reduce the incidence of recurrent VF, but also the duration of episodes of recurrent $V F$, once they have been initiated.

\section{Future developments}

Our findings suggest that recurrences should be prevented, but also that the duration of each episode must be a short as possible. For the first objective, it could be considered to administer amiodarone earlier than currently recommended, when it is indicated after the third episode of VF. We could consider administering amiodarone already immediately after the first defibrillation. Such a new strategy must be investigated, preferrably in a randomized trial between the old and the new strategy

previous Guidelines, issued in 2000. (5) We could clearly demonstrate that the probability of initiating VF was 18 times higher in the first two seconds after start of chest compressions than in the period prior to start of chest compressions. The close relation between start of chest compressions and refibrillation make a direct mechanico-electrical cause more likely than the expected reperfusion from chest compressions. Knowing the time it takes to build up the coronary perfusion pressure, it is unlikely that coronary flow of any significance has occurred at the moment of refibrillation.

\section{Can refibrillation be prevented?}

Clearly, chest compressions are needed when a perfusing rhyhm is lacking Not giving chest compressions is no reasonable option. Amiodarone proved effective in recurrent or persistent VF in OHCA, resulting in increased survival to admission to the hospital. $(6,7)$ However, our observations on the association between VF burden and survival were made in a period, when the administration of amiodarone was performed according to the Guidelines 2005. Clearly administration of amiodarone alone is not a sufficient answer,
He current Guidelines 2005 for resuscitation instruct to resume CPR immediately after a shock and continue CPR for 2 minutes and not to interrupt CPR for an analysis of the heart rhythm. The baseline distortion of the ECG caused by chest compressions make it very difficult to analyse the rhythm in monitored patients, while the rhythm analysis of an AED as currently available, also does not allow the rhythm to be analysed, because the algorithm cannot separate the baseline distortion from the true rhythm. New algorithms are being developed for manual defibrillators and AEDs that may allow continuous monitoring of the heart rhythm, while chest compressions continue and recommend defibrillation when refibrillation occurs. If such an algorithm can be proven to be reliable, we can consider to interrupt CPR briefly for a defibrillation shock as soon as refibrillation occurs and immediately resume chest compressions after that shock, while the algorthm again monitors the heart rhythm. Such a new technical solution requires testing in the clinical setting of OHCA before it can be accepted, but is a promising new strategy to improve outcome of OHCA and recurrent VF. 


\section{REFERENCES}

1. White RD, Russell JK. Refibrillation, resuscitation and survival in out-of-hospital sudden cardiac arrest victims treated with biphasic automated external defibrillators. Resuscitation 2002;55:17-23.

2. Van Alem AP, Post J, Koster RW. VF recurrence: Characteristics and patient outcome in out-of-hospital cardiac arrest. Resuscitation 2003;59:181-8.

3. Hess EP, White RD. Ventricular fibrillation is not provoked by chest compression during post-shock organized rhythms in out-of-hospital cardiac arrest. Resuscitation 2005;66:7-11.

4. Osorio J, Dosdall DJ, Robichaux RP Jr, Tabereaux PB, Ideker RE. In a swine model, chest compressions cause ventricular capture and, by means of a long-short sequence, ventricular fibrillation. Circ Arrhythm Electrophysiol 2008;1:282-9.

5. Berdowski J, Tijssen JG, Koster RW. Chest compressions cause recurrence of ventricular fibrillation after the first successful conversion by defibrillation in out-of-hospital cardiac arrest. Circ Arrhythm Electrophysiol 2010;3:72-8.

6. Dorian P, Cass D, Schwartz B, Cooper R, Gelaznikas R, Barr A. Amiodarone as compared with lidocaine for shock-resistant ventricular fibrillation. N Engl J Med 2002;346:884-90.

7. Kudenchuk PJ, Cobb LA, Copass MK,Cummins RO, Doherty RO, Fahrenbruch CE, et al. Amiodarone for resuscitation after out-of-hospital cardiac arrest due to ventricular fibrillation. N Engl J Med 1999;341:871-8. 